\title{
Good Clinical Practices toward Safe Blood Transfusion: A Study of Blood Transfusion Process and providing Suggestions for streamlining the Same
}

\author{
${ }^{1}$ Akash A Shrivastava, ${ }^{2} \mathrm{G}$ Somu, ${ }^{3} \mathrm{M}$ Dayananda
}

\begin{abstract}
Introduction: Wrong blood transfusion (BT) is a medical negligence. Every hospital must have a strong policy to check incorrect BT and see to it that these policies are strictly implemented at the time of transfusion.

Wrong BT can occur due to carelessness of the staff and shortcomings in verification of the blood bag. The reasons can be avoided and wrong BT can be prevented by the formation of a checklist consisting of the important details to be verified before initiating transfusion. The checklist should not be very long and time-consuming, but very comprehensive and consists of only absolutely essential things to be checked.
\end{abstract}

Aim: To study the BT process and providing suggestions for streamlining the process of BT.

Objectives:

- To analyze the nears miss incidents during BT.

- To identify the errors in the process of transfusion.

- To streamline the process by introducing checklist/work instructions for reducing errors.

\section{Materials and methods:}

- Analysis of safety reports regarding BT.

- Process-based root cause analysis was done at the time of issue and at ward level.

- Feedback regarding BT was taken from the staff working at blood bank and nursing professionals.

The study was divided into two phases:

Phase 1: January-April 2014

Phase 2: May-August 2014

All the reports from phase 1 of the study were analyzed. Based on the observations, interventions in the form of checklist and work instructions to the nursing staff were implemented in the hospital in the month of April and then the safety reports for the next 4 months were analyzed.

Interventions done: A " 4 C" checklist was created with just four elements that could be orally or mentally reviewed before beginning transfusion. Specific work instructions were also issued to the nursing staff at the ward level to prevent any errors during labeling of the samples being sent for cross match and blood grouping before BT.

\footnotetext{
${ }^{1}$ Resident, ${ }^{2}$ Head, ${ }^{3}$ Professor, Medical Superintendent and Chief Operating Officer

${ }^{1-3}$ Department of Hospital Administration, Kasturba Medical College, Manipal, Karnataka, India
}

Corresponding Author: Akash A Shrivastava, Resident Department of Hospital Administration, Kasturba Medical College, Manipal, Karnataka, India, Phone: +918202922587 e-mail: akash.anand2911@gmail.com
Results: The number of BT-related safety incidents observed in phase 1 reduced in phase 2 though the workload in terms of samples received remained comparable for the two phases. However, a declining trend for the reporting of incidents was also seen through the phases.

Keywords: Blood transfusion, Checklist, Process reengineering, Work instructions.

How to cite this article: Shrivastava AA, Somu G, Dayananda M. Good Clinical Practices toward Safe Blood Transfusion: A Study of Blood Transfusion Process and providing Suggestions for streamlining the Same. Int J Res Foundation Hosp Healthc Adm 2016;4(1):1-4.

\section{Source of support: Nil}

Conflict of interest: None

\section{INTRODUCTION}

"Wrong blood transfusion (BT) is an error, which no hospital/doctor exercising ordinary care would have made. Such an error is not an error of professional judgment but, in the very nature of things, a sure instance of medical negligence." ${ }^{\prime 1}$

Every hospital must have a strong policy to check incorrect BT and see to it that these policies are strictly implemented at the time of transfusion.

The recent testing facilities have lowered the incidence of transfusion-transmitted diseases to minimum; however, the incidence of adverse events due to human errors, ABO incompatibility, alloimmunization, bacterial contamination, and immunomodulation phenomena remain a matter of concern. ${ }^{2}$

This study focused only on avoidable human errors in ABO incompatible BT and no other hemolytic transfusion reaction (Table 1$){ }^{3}$

As Peter Drucker once said, "If you can't measure it you can't manage it." Thus, the importance of effective measurement in checking any error cannot be overstated.

The study encompasses measuring of errors in the BT process, which could lead to potential mismatch transfusions, analyzing the errors and introducing focused interventions to bring down such errors, thereby making the transfusion process safer with no room for error, be it human or otherwise. 


\begin{tabular}{|c|c|c|c|c|c|c|c|c|c|c|}
\hline \multirow[b]{2}{*}{ Year } & \multicolumn{2}{|c|}{2007} & \multicolumn{2}{|c|}{2008} & \multicolumn{2}{|c|}{2009} & \multicolumn{2}{|c|}{2010} & \multicolumn{2}{|c|}{2011} \\
\hline & No. & Percent & No. & Percent & No. & Percent & No. & Percent & No. & Percent \\
\hline $\begin{array}{l}\text { Hemolytic transfusion } \\
\text { reaction }\end{array}$ & 3 & 6 & 10 & 22 & 4 & 9 & 2 & 5 & 3 & 10 \\
\hline Total & 52 & & 46 & & 44 & & 40 & & 30 & \\
\hline
\end{tabular}

A "nearmiss" event refers to any error, which if undetected, could result in the determination of a wrong blood group or transfusion of an incorrect component, but was recognized before the transfusion took place. ${ }^{4}$

Definition of wrong blood in tube (WBIT) incidents ${ }^{5}$ :

- Blood is taken from the wrong patient and is labeled with the intended patient's details.

- Blood is taken from the intended patient, but labeled with another patient's detail.

\section{MATERIALS AND METHODS}

Study period: Eight months.

Study design: Prospective observational study.

Measurement of errors in the first half followed by implementation of policy in the form of checklist and work instructions in the second half and finally measuring the outcomes of the intervention.

- Analysis of safety reports regarding BT was carried out.

- Process-based root cause analysis was done at the time of issue and at ward level.

Data was collected from the Safety committee, Medical Records Department, and the Department of Immunohematology and BT.

Setting: Conducted at National Accreditation Board for Hospitals and Health care Providers (NABH) accredited 2,032-bedded tertiary care teaching hospital with in-house blood bank.

The Ethics Committee of the institution approved the study.

\section{DATA SOURCES}

- Incident reporting safety and sentinel forms.

- Feedback regarding BT taken from blood bank and nursing staff.

\section{Incident Reporting}

The hospital has a policy to report all patient safetyrelated incidents through a safety/sentinel form with the details of the incident. The staff involved in the incident initiates the form by filling the details of the affected party like name, hospital number, and ward along with the details of the initiator. The completely filled form is sent to the head of the department or the nursing superintendent depending on the department of the staff initiating the report (clinical or nursing). After perusal by the concerned authority, the form is sent to the Chairman of the Safety Committee. The Safety Committee conducts a root cause analysis of the report and suggests ways to prevent further incidents. The safety-related issues of the entire month are then compiled and presented in a safety meeting attended by medical superintendent, consultants of various departments, nursing in-charges, operations team, fire officer, security in-charge, and other staff.

For the study safety reports of 8 months from January to August 2014 were analyzed.

The study was divided into two phases:

Phase 1: January-April 2014

Phase 2: May-August 2014

All the reports from phase 1 of the study were analyzed. Based on the observations, interventions in the form of checklist and work instructions to the nursing staff were implemented in the hospital in the month of April and then the safety reports for the next 4 months were analyzed.

Meetings with all the stakeholders were called and initiatives to train the nursing staff focused on safe BT practices were taken.

\section{OBSERVATIONS}

Process of blood requisition from the wards/intensive care units and issue of blood component from the blood bank:

- Treating doctor decides the need for transfusion of blood component.

- Patient is explained about the need of transfusion and consent is taken.

- The treating doctor fills blood requisition slip.

- Patient sample for grouping and cross-match is drawn and sent to blood bank with the requisition slip.

- Requisition slip and sample of the patient are received at the counter in blood bank.

- All details are filled in the software and a Blood Bank Registration (BBR) number is generated.

- The sample of the patient is sent for cross-match and grouping and for other investigation.

- Blood bag of the same group is cross-matched, tested for compatibility, and kept ready for issue.

- On receival of issue slip from the ward, the blood is issued after checking for the details. 
Processes identified where errors occurred: Step 4 and 9.

Reasons for error at ward for sending WBIT for grouping/cross-match:

- Same name of more than one patient admitted in the ward.

- The sample of one patient might be sent with the labeling of other patient (interchanging of labels).

- The Hospital number/IP number of the patient was not checked before labeling of the sample.

- Labeling of empty tube was done before drawing the blood.

Reasons for near missed wrong BT in ward:

- Blood transfusion required by more than one patient in the ward.

- Proper instructions not conveyed to the junior nursing staff by the in-charge.

- Patient details not verified before starting transfusion. Reasons for error during issuing of the blood product from Blood Bank:

- The requisition slip of one patient might be sent with the blood product meant for some other patient.

- Blood/blood product dispatched from the Blood Bank for one patient maybe labeled for some other patient.

- The staff may not crosscheck the blood product before issue.

Process re-engineering:

Based on root cause analysis of safety reports, feedback of nursing and blood bank staff, interview with various stakeholders, and observation of workflow at the user level (wards) as well as at the dispatch level (blood bank), it was found that most of the errors took place in the wards.

Though a BT form in accordance with National AIDS Control Organization (NACO) guidelines with 18 entries like name and address of patient, IP number with blood group, blood unit received from blood bank, donor's ID number, reasons for transfusion, etc. is available in the wards, it failed to check the human error likely to take place before starting transfusion.

To address this problem a very short yet comprehensive checklist needed to be created, which would prevent wrong BT and still not add to the already overwhelming paperwork for the nursing staff.

Therefore a " $4 \mathrm{C}$ " checklist was created with just four elements that could be orally or mentally reviewed before beginning transfusion.

- Confirm - Hospital number

- Converse

- Consent

- Cross-match report
The nursing staff was first supposed to confirm the identity of the patient with the Hospital number and not the name of the patient. The issue slip, blood bag, and the cross-match slip would be tallied only with the hospital number of the patient.

It was stressed that conversing with a conscious and oriented patient was very essential before beginning transfusion. The patient would definitely know whether the doctor has advised transfusion for him/ her. Keeping the element of conversation in the checklist would also stress on the basic and essential yet easily missed component of patient involvement in treatment.

Consent of the patient is of utmost importance and any checklist should have this element as a necessity, for it is not only ethical but also a medico-legal requirement to check for consent. Checking of cross-match report will ensure that the patient is transfused the correct bag of blood. Thus, by having a checklist which was easy to remember and noncumbersome, the compliance in implementing the checklist among the nursing staff was ensured.

Specific work instructions were also issued to the nursing staff at the ward level to prevent any errors during labeling of the samples being sent for cross-match and blood grouping before BT. These were specific step-bystep instructions from checking the identity of the patient, drawing and labeling of the blood sample to sending the correct blood sample for investigation.

The work instructions would not only serve as a ready reckoner for the nursing staff but would also help in training of newly recruited staff.

The work instructions were as follows:

- Check the doctor's order and confirm that the patient requires $\mathrm{BT}$.

- Check the grouping/cross-match requisition slip for the hospital number.

- Check for consent of the patient.

- Select the proper vacutainers (lavender for grouping, red for cross-match).

- Go to the bedside with the file, vacutainers, and requisition slip.

- Talk to the patient and confirm whether the doctor has advised bt or not.

- Draw the sample and label after drawing the blood.

- Send the blood sample for investigation to blood bank.

The above-mentioned " $4 \mathrm{C}$ " checklist and work instructions for sending the sample for investigations were implemented in the tertiary care center at the end of phase 1 in the month of April and the outcome was measured in the next 4 months, i.e., phase 2 of the study. 


\begin{tabular}{ll}
\hline \multicolumn{1}{c}{ Table 2: Phase 1 } & \\
\hline Total no. of safety incidents reported & 34 \\
Transfusion-related incidents & 7 \\
Transfusion-related incidents reported from & 5 \\
blood banks & \\
Transfusion-related incidents reported from wards & 2 \\
Near missed wrong BT & 2 \\
Wrong blood in tube & 4 \\
Wrong labeling of blood product & 1 \\
Safety reports initiated by nursing staff & 2 \\
Safety reports initiated by other staff & 5 \\
No. of blood components issued & 9,873 \\
No. of cross-match samples received & 14,951 \\
\hline
\end{tabular}

\section{RESULTS}

The results of phase 1 and phase 2 are tabulated in Tables 2 and 3 respectively.

The number of BT-related safety incidents reduced from seven in phase 1 to two in phase 2. There were two incidences of near missed events of wrong BT during phase 1 and none during phase 2. The incidences of wrong labeling of sample or blood bag came down from five during phase 1 to two after the interventions were implemented in phase 2 . There was one incident of issue of product under incorrect label in phase 1 and none in phase 2.

There was not much difference in the number of cross-match samples received for testing by the blood bank during phase $1(14,951)$ and phase $2(15,399)$. The number of blood bags issued during the two phases was also comparable. It was 9,873 during phase 1 and 9,066 during phase 2 .

Total number of safety incidents too had shown a decreasing trend from 34 in phase 1 to 13 in phase 2. The number of safety incident reporting from nursing staff from the wards decreased from two in phase 1 to nil in phase 2 .

\section{CONCLUSION}

Analysis of safety and sentinel incident reports demonstrated various reasons for WBIT and near missed

wrong BT. Therefore, focused interventions in the form of checklist and work instructions were carried out which proved to be effective in reducing the number of human errors that could lead to wrong BT. However, the reporting of incidents also decreased probably due to the repeated interviews of staff by the authors. Though the intervention could not reduce the numbers to zero, which is desirable, it is expected that continuation of the practices initiated would, in time, achieve the desired results.

It was demonstrated that improvement in quality of health care can be brought about by need based, focused intervention and that analysis and measurement of errors were pivotal in reducing human errors.

\section{REFERENCES}

1. Postgraduate Institute of Medical Education \& Research, Chandigarh vs. Jaspal Singh \& Others, SC Civil Appeal no. 7950 of 2002; 2009 May 29.

2. Bhattacharya P, Marwaha N, Dhawan HK, Roy P, Sharma $R R$. Transfusion-related adverse events at the tertiary care center in North India: an institutional hemovigilance effort. Asian J Transfus Sci 2011 Jul-Dec;5(2):164-170.

3. Fatalities Reported to FDA Following Blood Collection and Transfusion. Annual Summary for Fiscal Year 2011. p. 1-15.

4. Watt A. Near Miss reporting (NM). Annual SHOT report 2013. p. 47.

5. Ball J, Mistry H, Gallagher C, Bolton-Maggs P. Incorrect blood component transfused (clinical and laboratory errors). Annual SHOT report 2013. p. 57. 